\title{
Phosphorylation and association with the transcription factor Atf1 regulate localization of Spc1/Sty1 stress-activated kinase in fission yeast
}

\author{
Frédérique Gaits, Geneviève Degols, Kazuhiro Shiozaki, ${ }^{1}$ and Paul Russel ${ }^{1,2}$ \\ Departments of Molecular Biology and Cell Biology, The Scripps Research Institute, La Jolla, California 92037 USA
}

\begin{abstract}
Control of gene expression by stress-activated protein kinase (SAPK) cascades is crucial for combating cytotoxic stress. Elements of these cascades have been investigated in detail, but regulation of stress signal transduction from the cytoplasm to the nucleus is poorly understood. Herein are reported subcellular localization studies of fission yeast Spc1, a homolog of human p38 and budding yeast Hoglp SAPKs. Stress induces transient nuclear localization of Spc1. Nuclear translocation of Spc1 is coupled with disassociation from its activator kinase Wisl. However, Spcl does not concentrate in the nucleus of $\Delta$ wisl cells; therefore Wis1 does not tether Spc1 in the cytoplasm. Unphosphorylatable forms of Spc1 are dispersed in the cytoplasm and nucleus, even in cells that also produce wild-type Spc1. Thus, Spc1 must be phosphorylated by Wis1 to localize in the nucleus. Nuclear retention of Spc1 requires Atf1, a transcription factor that is the key nuclear substrate of Spc1. Nuclear localization of Atf1 requires Pcr1, a heterodimerization partner of Atf1. These studies show that phosphorylation and association with Atf1 are required for nuclear localization of Spc1.
\end{abstract}

[Key Words: Atf1; fission yeast; nuclear local ization; Schizosaccharomyces pombe; Spc1; stress-activated protein kinase]

Received January 21, 1998; revised form accepted February 29, 1998.

The rapid integration of signals from the plasma membrane to the nucleus is crucial for the appropriate gene expression responses to a vast array of external stimuli (Karin 1994; Hill and Treisman 1995; Karin and Hunter 1995). Cumulative efforts of many laboratories over the past decade have led to the el ucidation of several signal ing pathways involved in the regulation of the gene expression in response to external stimuli. All of these pathways include three sequential protein kinases, the prototype being the mitogen-activated protein kinase (MAPK) cascade found in mammals (Seger and Krebs 1995). The cascade is composed of a MAPK, a serinethreonine kinase that is activated through phosphorylation at a pair of conserved threonine and tyrosine residues by a MAPK kinase (MAPKK or MEK), which is itself phosphorylated and activated by a MAPKK kinase (MAPKKK or MKK) (Marshall 1994; Cobb and Goldsmith 1995). The temporal regulation of the phosphorylation cascade, as well as the spatial distribution of the elements of the pathway, accounts for the rapid transmission of information from the membrane to the nucleus (Cano and Mahadevan 1995).

\footnotetext{
${ }^{1}$ Present address: Section of Microbiology, Division of Biological Sciences, University of Califomia, Davis, Califomia 95616 USA.

${ }^{2}$ Corresponding author.

E-MAIL prussell@scripps.edu; FAX (619) 784-2265.
}

Investigations of the subcellular distribution of MAPK cascades have focused on extracellular signal-regulated kinase (ERK) cascades, which respond primarily to proliferation and differentiation signals ( $\mathrm{N}$ ishida and Gotoh 1993). The M KK and MEK components of these cascades reside in the cytoplasm, whereas ERK transl ocates from the cytoplasm to the nucleus after it has been phosphorylated by MEK (Chen et al. 1992; Gonzalez et al. 1993; Lenormand et al. 1993; Zheng and Guan 1994). Cytoplasmic localization of MEK requires a nuclear exclusion signal (NES) located in the amino-terminal part of the protein (Fukuda et al . 1996). Translocation of ERK to the nucleus is apparently not dependent on ERK phosphorylation or activation, because ERK mutants that cannot be phosphorylated by MEK or are otherwise catalytical ly inactive transl ocate into the nucleus upon celIular activation (Sanghera et al. 1992; Seth et al. 1992; Lenormand et al. 1993). Thus, it appears that the cytoplasmic localization of ERK is dependent on its association with MEK, which acts as a cytoplasmic anchoring protein. U pon activation, ERK dissociates from MEK and translocates into the nucleus (Fukuda et al. 1997). However, ERK does not appear to have a nuclear localization signal (NLS); therefore, it is not known how ERK accumulates in the nucleus.

The extended MAPK family includes a group of protein kinases that are activated by cellular stress. These 
comprise the JNK and p38 stress-activated protein kinases (SAPKs) in mammals (Davis 1994; Kyriakis et al. 1994). SAPKs are activated by different types of signals such as UV irradiation, heat, inflammatory cytokines, oxidative stress, and high osmolarity (Derijard et al. 1994; Han et al. 1994; Rouse et al . 1994; Waskiewicz and Cooper 1995). Very little is known about the localization of these kinases or their upstream activators. UV was shown to stimulate nucl ear transl ocation of JNK1 (Cavigelli et al. 1995), whereas p38 was found both in the cytoplasm and the nucleus and this pattern was unaffected by stress (Raingeaud et al. 1995). However, as noted by Raingeaud and coworkers, a caveat with the p38 studies is that they relied on ectopic overexpression and thus may not accurately reflect the distribution of endogenous p38.

Coping with stress is an ancient and universal challenge for eukaryotic cells; therefore, it is not surprising that even simple organisms such as yeasts have SAPK cascades that are homologous to the p38 pathway. Of particular interest is the cascade containing the SAPK Spcl in the fission yeast Schizosaccharomyces pombe. Like its mammalian counterpart, Spcl is activated by a wide range environmental insults, including high osmoIarity, oxidative conditions, heat, UV, and starvation. Spc1, also known as Sty1 or Phh1 (Millar et al. 1995; Shiozaki and Russell 1995; Kato et al . 1996), is activated by the MEK homolog Wisl, which phosphorylates threonine-171 and tyrosine-173 of Spc1. Wis1 is activated by the MKK homolog Wis4, also known as Wik1 and Wak1 (Shi ozaki and Russell 1996; Wilkinson et al. 1996; Samejima et al. 1997; Shiozaki et al. 1997). Spc1 is negatively regulated by tyrosine phosphatases Pyp1 and Pyp2 via direct dephosphorylation of tyrosine-173 (Millar et al. 1995; Shiozaki and Russell 1995; Degols et al. 1996). Interestingly, the Wis4-Wis1-Spc1 kinase cascade is linked to $\mathrm{G}_{2}-\mathrm{M}$ cell cycle control. In optimal growth conditions $\mathrm{spcl}^{-}$mutants exhibit a moderate delay of the onset of mitosis that is greatly exacerbated upon stress (Shiozaki and Russell 1995). The only substrate known for Spc1 is the transcription factor Atf1, which is responsible for Spc1-dependent regulation of gene expression in response to various forms of stress, although atf1 mutants do not exhibit the mitotic delay phenotype of spcl cells (Shiozaki and Russell 1996; Wilkinson et al. 1996).

The discovery of a conserved stress response mechanism in fission yeast presents a unique opportunity to use genetic tools to investigate the spatial organization of a SAPK cascade. These studies are described in this report. These experiments were performed with wildtype and mutant proteins expressed at normal physiological levels and thus are not subject to the caveats and complications associated with the previous studies of mammalian ERK and SAPK cascades. At the most fundamental level our findings concur with the previous work of mammalian ERKs, in that they identify Spc1 nuclear translocation as the primary mechanism of signal transduction from the cytoplasm to the nucleus. However, our studies present an entirely different pic- ture of how nuclear translocation of an SAPK is regulated.

\section{Results}

Nuclear translocation of Spc1 induced by stress

The subcellular distribution of Spcl was examined by indirect immunofluorescence microscopy. These studies utilized strains in which the chromosomal copy of spc1 encoded a protein containing 12 tandem copies of the myc epitope (see M aterials and Methods). These cells appeared identical to wild-type, therefore, the function of Spc1 was not impaired by the epitope tag. The myctagged Spc1 was detected using anti-myc monoclonal antibodies. Control experiments with wild-type cells encoding untagged Spc1 revealed no staining (Fig. 1A). Before stress, Spc1 was present in both the cytoplasm and nucleus, al though in most cells the nuclear signal was weaker (Fig. 1A). Osmotic stress $(0.6 \mathrm{M} \mathrm{KCl})$ caused rapid relocalization of Spcl into the nucleus. $\mathrm{N}$ ucl ear accumulation of Spcl was apparent within 5 min (F. Gaits and P. Russell, unpubl.) and maximal after $\sim 10$ min of stimulation (Fig. 1A). The nuclear accumulation of Spc1 was remarkably transi ent. Spc1 was quite obviously excluded from the nucleus within $20 \mathrm{~min}$ after the initial exposure to stress (Fig. 1A). Evaluation of Spc1 activation, as assayed by tyrosine phosphorylation, revealed that activation paralleled nuclear localization, with maximum ac-

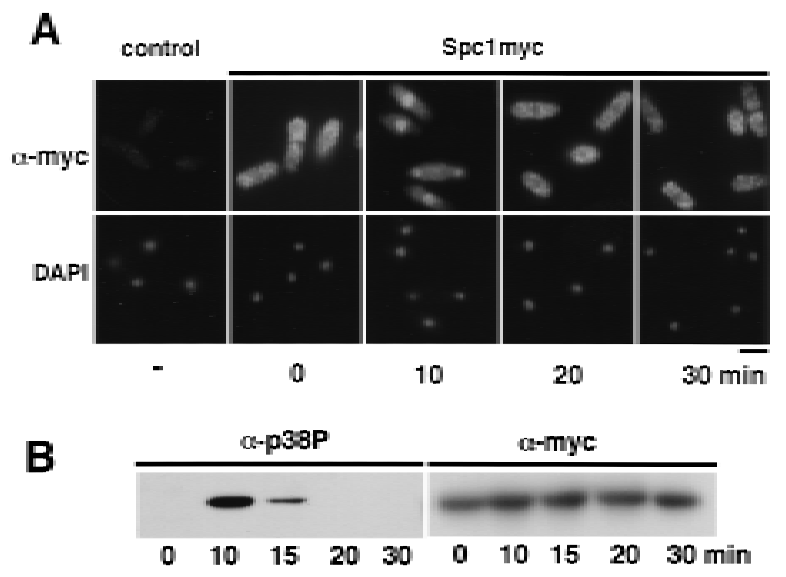

Figure 1. Spc1 localization with stress. (A) Spc1 translocates to the nucleus upon stress. Strain GD1942, in which the genomic copy of spc $1^{+}$encodes an epitope-tagged form of Spc1 that has 12 copies of a myc tag at the carboxyl terminus, was grown to mid-log phase at $30^{\circ} \mathrm{C}$ in YES medium. Aliquots were harvested before and after osmostress in YES $+0.6 \mathrm{M} \mathrm{KCl}$ at the indicated time and fixed in $-80^{\circ} \mathrm{C}$ methanol. After permeabilization, cells were incubated with the anti-myc antibody and Cy3 goat antimouse as a secondary antibody to visualize Spclmyc. The nuclei were stained with DAPI. Bar, $10 \mu \mathrm{m}$. (B) In parallel, some al iquots were frozen in liquid nitrogen and total cellular homogenates were prepared to evaluate Spc1 phosphorylation. Proteins were subjected to SDS-PAGE and electroblotted, and immunodetection of Spc1 was achieved using the anti-p38 phosphorylated antibody ( $\alpha$-p38P). The level of proteins in each lane was evaluated using the anti-myc antibody ( $\alpha$-myc). 


\section{Gaits et al.}

tivation occurring at $10 \mathrm{~min}$ followed by dephosphorylation within $20 \mathrm{~min}$ (Fig. 1B). These findings suggest that stress-stimulated nuclear transl ocation of Spcl is a major mechanism by which stress signal s are transduced from the cytoplasm to the nucleus.

Wis1, the kinase that activates Spc1, is located in the cytoplasm

Wisl is the sole activator kinase of Spcl in vivo. We therefore determined the localization of Wisl. This study used a strain that expressed myc-tagged Wisl from the wis $^{+}$genomic locus. This strain appeared identical to wild-type; therefore, the epitope tag had no effect on Wisl function in vivo. Wisl localized in the cytoplasm; no significant Wisl signal was detected in the nucleus before or during osmotic stress (Fig. 2A). These observations suggest that Wisl may be actively excluded from the nucleus. Wisl has several sequences that closely match the NES recently characterized in a mammalian MEK.

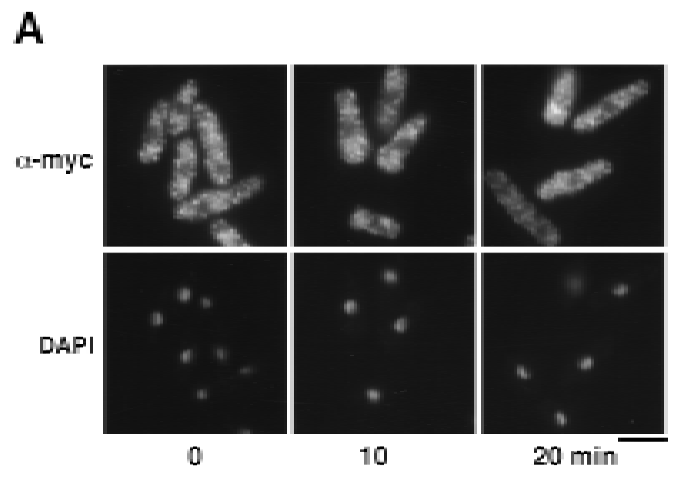

B

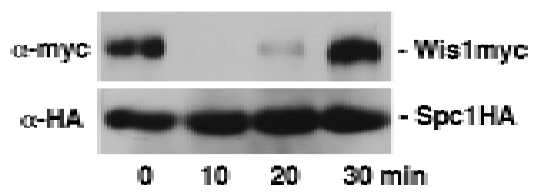

Figure 2. (A) Localization of Wisl MAPKK. Strain GD1892, bearing a genomic copy of wis1 ${ }^{+}$tagged with 12 copies of the myc epitope at the carboxyl terminus, was grown to mid-log phase at $30^{\circ} \mathrm{C}$ in YES medium. Cells were harvested before and after stress in YES $+0.6 \mathrm{M} \mathrm{KCl}$ at the indicated time and fixed in cold methanol. Wisl was visualized by immunofluorescence using anti-myc antibody. Bar, $10 \mu \mathrm{m}$. (B) Strain FG2150 bearing a genomic copy of $\mathrm{spcl}^{+}$tagged with two copies of the HAepitope and six consecutive histidine residues on its carboxyl terminus, as well as a genomic copy of wis $1^{+}$tagged with 12 copies of the myc epitope, was grown to mid-log phase and harvested before and after $\mathrm{KCl}$ stress at the indicated times. Total cell homogenates were then prepared under native conditions, and $\mathrm{Spcl}$ was purified using $\mathrm{Ni}^{2+}-\mathrm{NTA}$-agarose beads. The precipitates were analyzed by immunoblotting after SDSPAGE. Wisl was detected with the anti-myc and Spcl with anti-HA epitope antibodies.
Nuclear relocation of Spcl correlates with disassociation from Wisl in the cytoplasm

Next we investigated physical interactions involving Wisl and Spcl. We constructed a strain bearing a chromosomal copy of wisl expressing myc-tagged Wisl protein and a chromosomal copy of spcl expressing Spc1 tagged with the HA epitope followed by six histidine residues. Spc1HA6his was purified under native conditions with $\mathrm{Ni}^{+}-\mathrm{NTA}$-agarose. Wisl co-precipitated with Spcl before stress (Fig. 2B). U pon activation of the pathway by osmotic stress, the amount of Wisl associated with Spc1 decreased dramatical ly. This change was quite transient with Wisl becoming fully reassociated with Spcl within 30 min of the initial stress. This process paralleled the changes in Spcl phosphorylation and local ization (Fig. 1). These findings indicate that the affinity between Spc1 and Wisl is reduced in response to stress. The decreased association between the two proteins may allow translocation of Spcl into the nucleus.

\section{Spcl is predominantly cytoplasmic in $\Delta$ wisl cells}

Our findings were consistent with a model in which Wisl acts as a cytopl asmic anchor for Spc1. In this model Spcl has a tendency to localize in the nucleus, perhaps because of an interaction with another protein that transports Spc1 into the nucleus. Stress causes Spc1 to be released from Wisl, allowing translocation of Spc1 into the nucleus. This model makes a number of important predictions. One is that there should be roughly equal amounts of Wisl and Spc1, assuming that each Wisl protein interacts with a single Spcl protein. This prediction was tested by performing immunoblots of strains that expressed myc-tagged Spcl and Wisl from their respective genomic loci. This analysis reveal ed that $\mathrm{Spcl}$ is much more abundant than Wisl (Fig. 3A). These findings are inconsistent with a simple mechanism of cytoplasmic retention of Spc1 by Wisl, although they cannot exclude a more complex model in which each Wisl protein anchors many Spcl proteins.

A more definitive prediction of the model is that elimination of Wisl should cause Spcl to concentrate in the nucleus. We therefore investigated the behavior of Spcl in a strain in which wisl was deleted. Contrary to the model, we found that Spc1 was evenly distributed between the nucleus and cytoplasm in a $\Delta$ wisl mutant (Fig. 3B). This pattern of Spcl localization was unchanged following exposure to osmotic stress (Fig. 3B). Consistent with previous studies, no tyrosine phosphorylation of Spc1 was detected in the $\Delta$ wisl mutant (Fig. 3C). These findings argue against a model in which the cytoplasmic localization of Spcl is dependent on Wisl acting as a cytoplasmic anchor.

Activating phosphorylation is necessary for nuclear accumulation of Spc1

Our findings showed that Wisl activity is required for stress-induced nuclear rel ocation of Spc1. Like all of the 
A

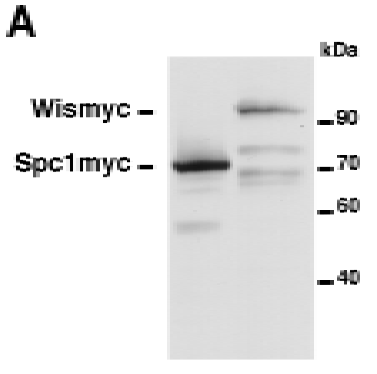

B

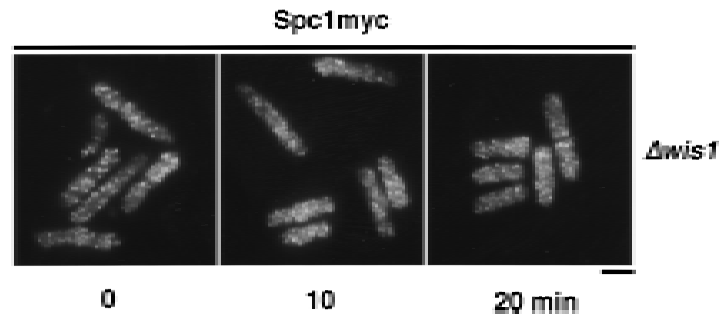

Figure 3. Wisl activity is necessary to translocate Spcl to the nucleus. (A) Spc1 is more abundant than Wisl. Total lysate of strains bearing myc-tagged genomic copies of Spcl or Wisl, respectively, strains GD 1942 and GD 1892, were prepared and proteins separated by SDS-PAGE. The levels of Spcl and Wisl in the extract were analyzed by immunoblotting with the antimyc antibody, the loading control being realized by amidoblack staining of the membrane prior to blotting. (B) Strain FG2151 (bearing the myc-tagged genomic copy of $\mathrm{Spc}^{+}$and deleted for wisl) was grown to mid-log phase in YES medium and cells were harvested before and after stress with $0.6 \mathrm{M} \mathrm{KCl}$. They were fixed in cold methanol, and Spcl localization was examined by immunofluorescence using the anti-myc antibody. Bar, $10 \mu \mathrm{m}$. (C) Aliquots of cells were collected before and after $\mathrm{KCl}$ stress, and the phosphorylation status of Spcl was analyzed by immunoblotting with the anti-p38P after SDS-PAGE. The loading control was realized by probing the membranes with antimyc antibody.

members of the greater MAPK family, Spc1 possesses conserved threonine and tyrosine resi dues that are phosphorylated by its cognate MEK. Wisl phosphorylates threonine-171 and tyrosine-173 in the motif TGY (M illar et al. 1995; Shiozaki and Russell 1995). To investigate further the role of these phosphorylations in regulating the localization of Spc1, three spcl mutations were engineered to encode the following proteins: Spc1AY (T171A), Spc1TF (Y173F), and Spc1AF (T171A, Y173F). Chromosomal replacements were performed with the myc-tagged spcl mutant genes, and the resulting phenotypes evaluated. Cells were streaked on standard YES (yeast extract and glucose) medium or medium supplemented with $1 \mathrm{~m} \mathrm{KCl}$. Wild-type, $\Delta$ spcl, and the mutants expressing unphosphorylatable forms of Spc1 all grew well on YES medium. However, on YES + KCl the spclAY, spclTF, spclAF, and $\Delta$ spcl mutants exhibited strong osmotic sensitivity and were unable to form colonies (Fig. 4A). Microscopic observation showed that the spc1AY, spc1TF, and spc1AF mutants displayed an elongated morphology phenotype that was identical to $\Delta \mathrm{spc} 1$ cells (Fig. 4B). Interestingly, Spc1AY became phosphory- lated on tyrosine in response to stress, showing that the T171A mutation did not disrupt interaction with Wisl (Fig. 4C). These findings confirm that Spcl activation requires phosphorylation on both threonine-171 and tyrosine-173.

Next we determined the localization of Spc1AY, Spc1TF, and SpclAF. These proteins failed to accumulate in the nucleus after osmotic stress (Fig. 4D), even at late time points (F. Gaits and P. Russell, unpubl.). The localization pattern of the mutant Spcl proteins appeared identical to wild-type Spcl in $\Delta$ wisl cells (Fig. 3B). These findings show that Wisl-catalyzed phosphorylation on both threonine-171 and tyrosine-173 is somehow required for nuclear localization of Spc1.

Restoration of Spcl activity does not rescue the localization defect of unphosphorylatable Spc1

One interpretation of our data was that Spcl activity is required for nuclear translocation. For example, Spc1 may phosphorylate a protein that promotes nuclear transl ocation of Spc1. An al ternative explanation is Spc1 must be phosphorylated on threonine-171 and tyrosine173 to localize in the nucleus. For example, phosphorylation of these residues may promote association with a nuclear transport system or stabilize interaction with a nuclear anchor. To distinguish between these hypotheses, the localization of mutant Spcl was monitored in heterozygous diploid strains containing mutant and wild-type spcl alleles. A prediction of the first hypothesis is that restoration of Spcl activity should rescue the localization defect of unphosphorylatable Spc1. Conversely, the second hypothesis predicts that unphosphorylatable forms of Spcl should be unable to concentrate in the nucleus of cells that express wild-type Spc1.

Diploid strains having one copy of wild-type $\mathrm{spc}^{+}$and one copy of the mutant genes were not sensitive to osmotic stress when compared to homozygous wild-type diploid strains (Fig. 5A), indicating that the spc1AY, spc1TF, and spc1AF alleles behaved as typical recessive mutations and the stress-activated kinase cascades were fully operable. In the homozygous wild-type diploid $\left(\mathrm{spc1}{ }^{+} / \mathrm{spcl}^{+}\right.$:myc), myc-tagged Spcl concentrated in the nucleus in response to osmotic stress (Fig. 5B). In contrast, in the heterozygous diploids containing one copy of myc-tagged mutant spcl and one copy of the wild-type spc $1^{+}$, the mutant forms of Spc1 failed to accumulate in the nucleus in response to stress (Fig. 5B). These data establish that Spc1 must be phosphorylated on threonine-171 and tyrosine-173 to accumulate in the nucleus.

\section{Transcription factor Atf1 is a nuclear anchor for Spc1}

Having established the requirement of Wisl-catalyzed phosphorylation for nuclear localization of Spc1, we then turned our attention to the role of the transcription factor Atf1, a key substrate of Spcl (Shi ozaki and Russell 1996; Wilkinson et al. 1996). First, using the strategies described above, we determined the local ization of Atf1. The myc-tagged Atf1 was constitutively localized in 
A

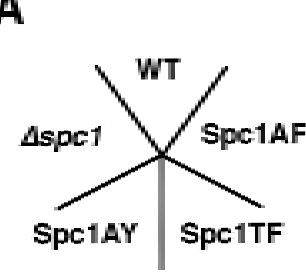

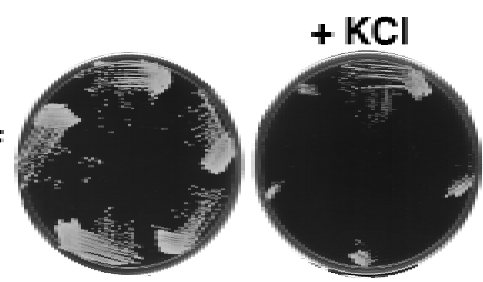

B

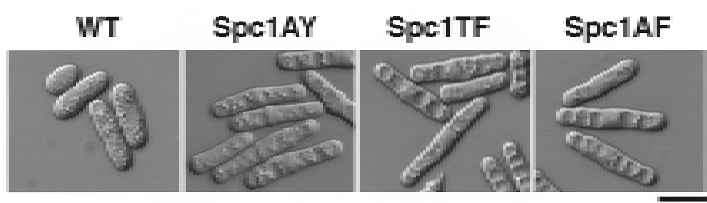

C

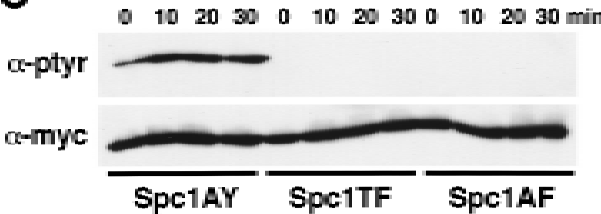

D
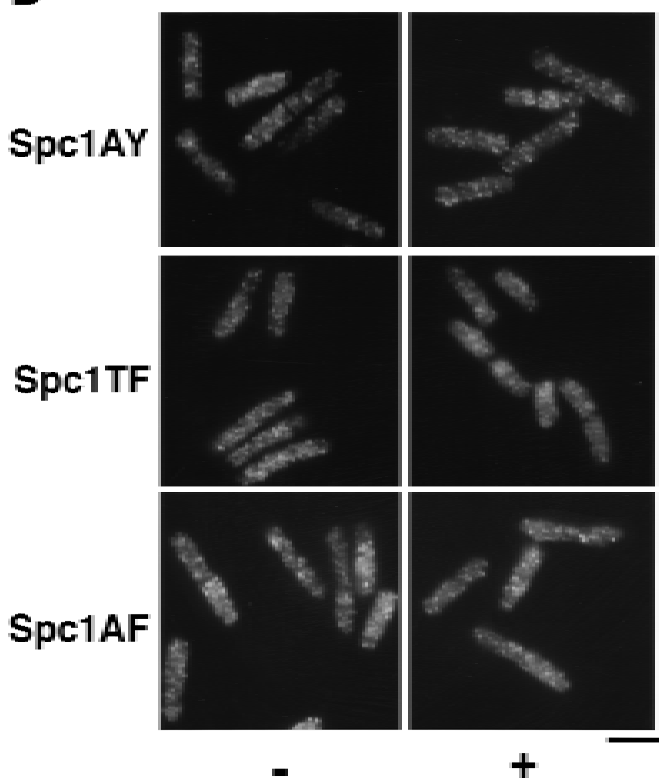

Figure 4. Spcl phosphorylation mutants are sensitive to high osmolarity and unable to relocate to the nucleus upon osmotic stress. (A) Strains FG2153 (genomic spc1+ replaced by spc1T 171A myc), FG2154 (genomic spc1 ${ }^{+}$replaced by spc1Y 173Fmyc), FG2155 (genomic spc1 $1^{+}$replaced by spc1T171A Y173Fmyc), KS1366 ( $\Delta$ spc1), and PR109 (wild type) were streaked out onto YES agar plates supplemented with or without $1 \mathrm{M} \mathrm{KCl}$, and incubated for 4 days at $32^{\circ} \mathrm{C}$ to test their ability to form colonies. (B) FG2153, FG2154, FG2155, and PR109 cells were streaked out on EM M 2 agar plates. The phenotype of the cells was then analyzed by phase contrast microscopy after 2 days at $32^{\circ} \mathrm{C}$. Bar, $10 \mu \mathrm{m}$. (C) The Spc1 mutant strains FG2153, FG2154 and FG2155 were grown to mid-log phase in YES medium and stressed with $0.6 \mathrm{M} \mathrm{KCl}$ before being harvested and frozen in liquid nitrogen. Total lysates were then prepared, and the phosphorylation status of the Spcl mutants was analyzed by immunoblotting after SDS-PAGE using the anti-phosphotyrosine antibody ( $\alpha$-pTyr). The loading was checked using the anti-myc antibody ( $\alpha$-myc). (D) Strains FG2153 (Spc1AY), FG2154 (Spc1TF), FG2155 (Spc1AF) were grown to mid-log phase in YES medium, harvested before or after 10 min of incubation in YES supplemented by $0.6 \mathrm{M} \mathrm{KCl}$, and fixed in cold methanol. The cellular localization of the mutant Spcl was followed by indirect immunofluorescence with the anti-myc antibody. Bar, $10 \mu \mathrm{m}$.

the nucleus, both before and during exposure to osmotic stress (Fig. 6A). As al ready shown at the transcriptional level (Shiozaki and Russell 1996; Wilkinson et al. 1996), immunofluorescence indicates that the quantity of Atf1 increases after $\mathrm{KCl}$ treatment (Fig. 6A). We then examined the localization of Spc1 in cells lacking Atf1. Osmotic stress failed to induce the accumulation of Spcl in the nucleus of $\Delta$ atf1 cells (Fig. 6B). Importantly, Spc1
Figure5. Restoration of Spcl activity does not rescue the localization defect of the unphosphorylatable mutants. (A) The haploid strains FG2153 (Spc1AY), FG2154 (Spc1TF), and FG2155 (Spc1AF), and the diploid strains FG2201 (Spc1WT/WT), FG2202 (Spc1AY/WT), FG2203 (Spc1TF/WT), and FG2204 (Spc1AF/WT) were streaked out onto YES agar plates supplemented with 1 $\mathrm{M} \mathrm{KCl}$ and incubated for 3 days at $32^{\circ} \mathrm{C}$. The phenotype of the cells was then analyzed by phase-contrast microscopy. (B) The diploid strains FG2201 (Spc1WT/WT), FG2202 (Spc1AY/WT), FG2203 (Spc1TF/WT), and FG2204 (Spc1AF/WT) were grown to midlog phase in YES medium. Cells were then harvested before and after $10 \mathrm{~min}$ of $\mathrm{KCl}$ stress and fixed with cold methanol. The localization of the Spcl proteins was then determined as described previously. Bar, 10 $\mu \mathrm{m}$.

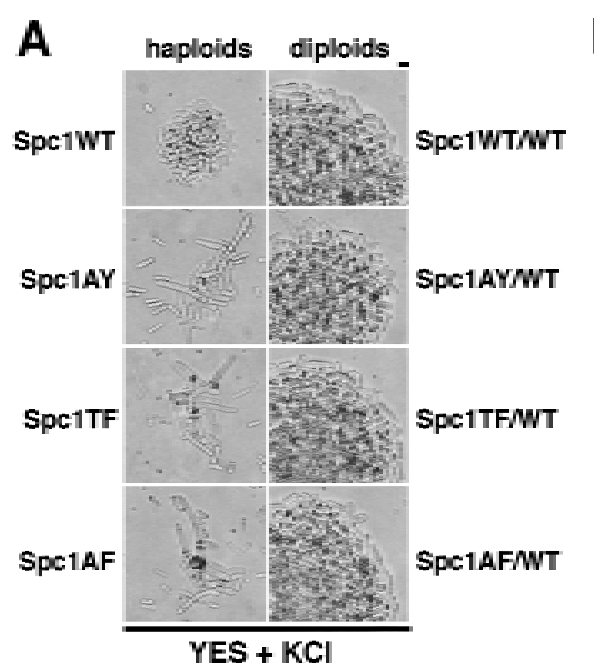

B

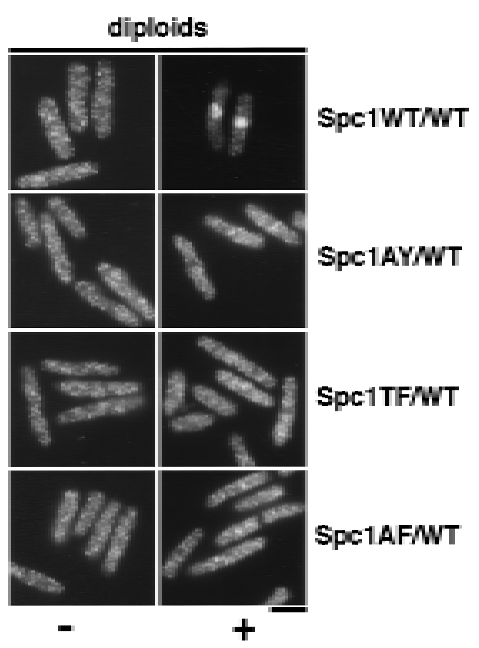




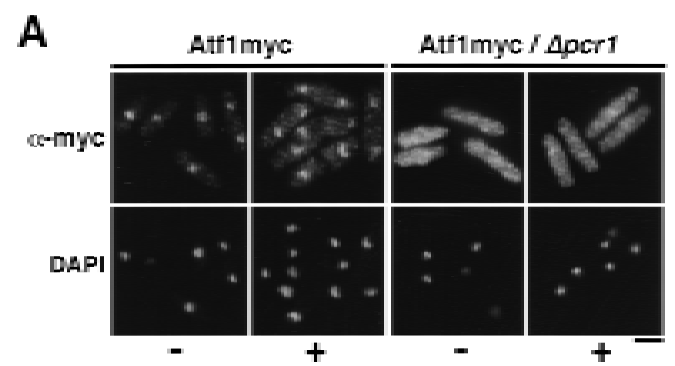

B
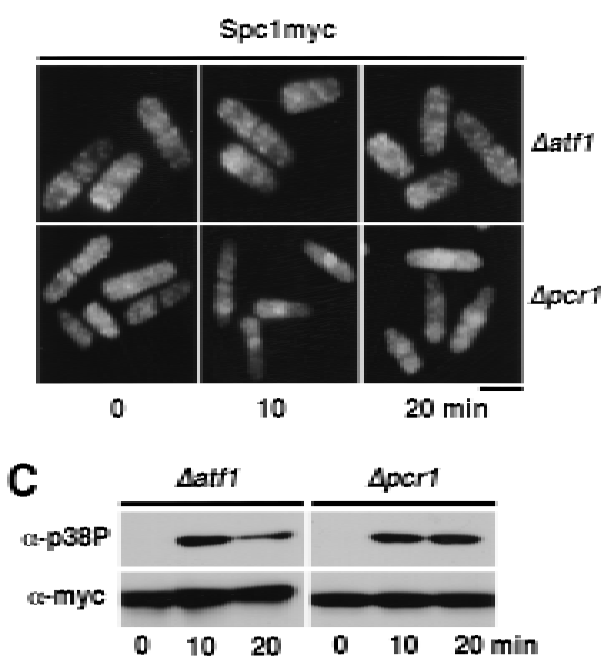

Figure 6. Atf1 acts as a nuclear anchor for $\operatorname{Spcl}$. (A) The localization of Atf1 was determined by immunofluorescence performed on strain FG2156 in which the genomic atf $1^{+}$is replaced by a myc-tagged copy, and in the FG2157 ( $\Delta$ pcrl and bearing the genomic myc-tagged atf1 ${ }^{+}$). Cells were harvested before and after $10 \mathrm{~min}$ of $\mathrm{KCl}$ stress and fixed in cold methanol. The immunofluorescence was real ized using the anti-myc antibody. (B) The strain GD 1952 ( $\Delta$ atf1 and bearing the genomic myc-tagged copy of $\left.\mathrm{spc}^{+}\right)$and $\mathrm{FG} 2158(\Delta \mathrm{pcr} 1$ and bearing the myc-tagged genomic $\mathrm{spcl}^{+}$) were grown to mid-log phase, and cells were harvested before and after $\mathrm{KCl}$ stress. For each time point, an aliquot was fixed in cold methanol for immunofluorescence analysis. The local ization of Spc1 was determined with the antimyc antibody. (C) The phosphorylation status of Spcl in strains GD1952 and FG2158 was followed with the anti-p38P and the loading checked with the anti-myc antibodies, as described previously. Bar, $10 \mu \mathrm{m}$.

became tyrosine-phosphorylated in $\Delta$ atfl cells at a level comparable with wild type (Fig. 6C). These findings are most simply interpreted to indicate that the transient nuclear local ization of activated Spcl is dependent on an association with Atf1.

\section{Nuclear localization of Atf1 requires its cofactor Pcr1}

Atf1 forms a heterodimer with the transcription factor Pcr1 (Watanabe and Yamamoto 1996). $\Delta$ pcr1 and $\Delta$ atf1 strains exhibit similar levels of stress sensitivity, and $\Delta$ pcrl cells are defective in the expression of most genes that are dependent on Atf1 for expression. In an attempt to gain some initial insights into the mechanism by which Atf1 is retained in the nucleus, we investigated whether Pcrl was required for the nuclear accumulation of Atf1. In $\Delta$ pcrl cells, Atf1 was equally distributed between the nucleus and cytoplasm (Fig. 6A). As was the case for $\Delta$ atf1 cel Is, Spcl was tyrosine phosphorylated in $\Delta$ pcrl cells (Fig. 6C). The phosphorylation of Spc1 was slighty prolonged, reflecting the difficulty of cells to adapt to stress due to the defect of transcription of stress-response genes (Shiozaki and Russell 1996; Wilkinson et al. 1996). Nuclear accumulation of Spcl was greatly reduced in $\Delta$ pcrl cells, although a weak nuclear signal was detected in some cells during osmotic stress (Fig. $6 \mathrm{~B})$. These findings suggest that Atf1 nuclear retention requires dimerization with Pcrl. Moreover, the presence of Atf1 is not in itself sufficient to localize Spc1 in the nucleus because Spcl is excluded from the nucleus after stress when the quantity of Atf1 is maximal (Figs. 1A and $6 \mathrm{~A}$ ). Instead, after activation of the cascade, Atf1 is required in the nucleus for nuclear retention of Spc1.

\section{Discussion}

Our studies have expl ored the spatial regulation of three sequential elements of the stress signal transduction system: The kinases Wisl and Spcl and the transcription factor Atf1. We have established that Wisl is detected completely in the cytoplasm, whereas Atf1 is found exclusively in the nucleus. These patterns of Iocalization are unaffected by stress. In contrast, stress induces a rapid relocalization of Spc1 from the cytoplasm to the nucleus. Spc1 nuclear import closely coincides with activation of Spcl as assayed by tyrosine phosphorylation, as well as with transcriptional induction of stress response genes. These observations suggest strongly that the rapid nucl ear import of activated Spcl is the primary mechanism by which the stress signal is transmitted from the cytoplasm to the nucleus.

We have also addressed the mechanism regulating the localization of Spc1. Our studies have tested the hypothesis that Wisl acts as a cytoplasmic anchor for Spcl. Generation of this hypothesis was based on studies of vertebrate ERKs. In mammalian cells these kinases are translocated into the nucleus following growth factor stimulation (Sanghera et al. 1992; Seth et al. 1992; Lenormand et al. 1993). However, high expression of ERKs, accomplished either by plasmid transfection or by protein microinjection, leads to nuclear localization by a mechanism that is independent of growth factors. $\mathrm{N}$ uclear localization of ERKs was prevented by microinjection of large amounts of a protein containing the NES and MAPK-binding region of a cognate MEK (Fukuda et al. 1997). These findings led to the hypothesis that MEK is required to retain inactive ERK in the cytoplasm. This hypothesis generates a number of predictions, the most crucial being that elimination of cognate MEKs should cause nuclear localization of ERKs. This prediction is not easily tested with mammalian cells, but the genetical advantages of fission yeast provided an ideal system for testing the model as it pertains to the SAPK cascade. Contrary to the key prediction of the MEK-anchor model, we found that Spcl was distributed throughout 
the cell in $\Delta$ wisl strains. Spcl did not appear to be excluded from the nucleus, but the majority of the Spc1 protein was in the cytoplasm. Thus, in unstressed cells, the cytoplasmic local ization of the majority of Spc1 protein does not depend on an interaction with Wisl. It is very unlikely that Spcl is anchored in the cytoplasm through interactions with another M APKK homolog, because Spcl tyrosine phosphorylation is abolished in a $\Delta$ wisl cell.

How is Wisl required for nuclear accumulation of Spc1? Wisl activates Spcl by phosphorylating threonine171 and tyrosine-173; therefore, we asked whether nuclear accumulation of Spcl requires phosphorylation of these sites. We found that Spc1 mutant proteins that had substitutions at either or both phosphorylation sites failed to concentrate in the nucleus in response to stress. The Spc1 localization patterns of these mutants were very similar to the staining pattern of Spcl protein in $\Delta$ wisl cells. These findings led to the obvious conclusion that Spcl protein fails to accumulate in the nucleus of $\Delta$ wisl cells because it cannot be phosphorylated.

Phosphorylation of threonine-171 and tyrosine-173 is required for $\mathrm{Spc1}$ kinase activity, suggesting that Spc1 kinase activity is required for nuclear import of Spcl. In such a scenario one could propose that a substrate of Spcl is involved in regulating the nuclear import of Spcl. If this model was correct, we would have expected that nuclear import of the Spc1AY, Spc1TF, and Spc1AF mutants be restored by coexpression of active Spc1. However, our studies showed clearly that this prediction was incorrect. The mutant forms of Spcl did not accumulate in the nucleus of diploid cells in which half of the Spc1 was expressed from a wild-type copy of $\mathrm{spcl}^{+}$, even though these cells appeared wild type in terms of cell size and sensitivity to osmotic stress. These findings led to the conclusion that Spcl protein must be phosphorylated on threonine-171 and tyrosine-173 to localize in the nucleus. This fact contrasts sharply with studies of MAPK localization in mammalian cells, which have shown that unphosphorylatable forms of ERK2 localize in the nucleus, at least when they are overexpressed (Lenormand et al. 1993).

Why is phosphorylation of threonine-171 and tyrosine173 essential for nuclear localization of Spc1? One possible explanation is that phosphorylation of these sites stabilizes an interaction of Spc1 with a nuclear anchor protein. Atf1 is a key substrate of Spcl and is localized in the nucleus; therefore, we asked whether Atf1 was required for nuclear localization of Spcl. We found that nuclear accumulation of Spc1 was abolished in an $\Delta$ atf1 strain, showing that Atf1 is required for retention of Spc1 in the nucleus. In this sense Atf1 is a nuclear anchor for activated Spcl. These findings suggest a model in which Wisl-catalyzed phosphorylation of Spc1 enhances the ability of Spc1 to associate with Atf1. Thus, it suggests that the stabilized association of phosphorylated Spc1 with Atfl causes the nuclear accumulation of Spcl.

Little is known about the role of activating phosphorylation of MAPK homologs in regulating association with substrate transcription factors. There is no evidence sug- gesting that phosphorylation stimulates substrate binding; many of the in vitro studies performed to identify interaction domains have been carried out with unphosphorylated MAPK homologs. However, a careful analysis of the effect of phosphorylation on binding affinities has not been reported. Studies of the mammalian JN K1 and JNK2 stress-activated kinases identified a small region near the catalytic pocket that accounted for a $\sim 25$-fold difference in binding affinity for the amino-terminal domain of c-Jun (Kallunki et al. 1994). However, structure determinations of the dephosphorylated and phosphorylated forms of ERK2 suggest that the binding groove of JN Ks does not change as a consequence of phosphorylation (Canagarajah et al. 1997; Wang et al. 1997). These observations have prompted speculation that transcription factors such as c-Jun should bind to cognate SAPKs independent of the state of phosphorylation. In our system, the phosphorylation mutants Spc1AY, Spc1TF, and SpclAF associate with the GST-Atf1 fusion protein in vivo ( $F$. Gaits and P. Russell, unpubl.), indicating that phosphorylation of Spc1 is not required for binding to Atf1.

An alternative interpretation of our observations is that phosphorylation of threonine-171 and tyrosine-173 stimulates nuclear translocation of Spc1, at which point Atfl is required to retain Spcl in the nucleus. In this model phosphorylation might be required for (1) release of Spc1 from a cytoplasmic anchor; (2) association of Spc1 with a nuclear import protein; or (3) shielding of Spcl from a nuclear export system. The first explanation appears unlikely because Spcl does not localize in the nucleus when highly overexpressed in $\Delta$ wisl cells (F. Gaits and P. Russell, unpubl.); therefore, a cytoplasmic anchor, if it exists, cannot be saturated by very large amounts of Spcl. The other explanations cannot be excluded and thus deserve attention in future studies.

These studies raise an additional important point. The stress-induced relocalization of Spcl from the cytoplasm to the nucleus can be very transient, with Spcl becoming quite clearly absent from the nucleus 10-20 min after the peak nuclear signal is detected. This change correlates closely with the tyrosine dephosphorylation of Spcl and the reduction of stress-induced gene expression. These observations raise the question of the connection between export of Spcl from the nucleus and dephosphorylation of Spcl. Export of Spc1 from the nucleus may lead to dephosphorylation by cytoplasmic phosphatases. Alternatively, dephosphorylation of Spc1 by nuclear phosphatases may induce the export of Spc1 from the nucleus. Experiments to clarify the interrelation between phosphatase activity and Spcl relocalization are currently under way.

\section{Materials and methods}

Media, strains, and general techniques

S. pombe strains used in this study are listed in Table 1 . They are derivatives of $972 \mathrm{~h}^{-}$and $975 \mathrm{~h}^{+}$(Mitchison 1970). Growth media and basic genetic and biochemical techniques for fission yeast have been described (Alfa et al. 1993). Yeast extract me- 
Table 1. S. pombe strains used in this study

\begin{tabular}{|c|c|c|}
\hline Strains & Genotype & Source or reference \\
\hline PR109 & $\mathrm{h}^{-}$ & lab stock \\
\hline KS1366 & $\mathrm{h}^{-} \mathrm{spc1::ura4^{+ }}$ & Shiozaki and Russell (1995) \\
\hline GD1942 & $\mathrm{h}^{-}$spc1-12 myc (ura4+) & this study \\
\hline GD1952 & $\mathrm{h}^{-}$spc1-12 myc (ura4 ${ }^{+}$) atf1::ura4 ${ }^{+}$ & this study \\
\hline GD1892 & $\mathrm{h}^{-}$wisl-12 myc (ura4 $4^{+}$) & this study \\
\hline FG2150 & $\mathrm{h}^{-} \mathrm{spc} 1 \mathrm{HA} 6 \mathrm{H}\left(\mathrm{ura}^{+}\right)$wisl-12 myc (ura4 $\left.{ }^{+}\right)$ & this study \\
\hline FG2151 & 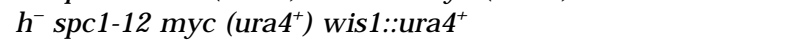 & this study \\
\hline FG2153 & $\mathrm{h}^{+}$spc1AY-12 myc (ura4 $\left.{ }^{+}\right)$ & this study \\
\hline FG2154 & $\mathrm{h}^{+}$spc1TF-12 myc (ura4+) & this study \\
\hline FG2155 & $\mathrm{h}^{+}$spc1AF-12 myc (ura4 $\left.{ }^{+}\right)$ & this study \\
\hline FG2156 & $\mathrm{h}^{-}$atf1-12 myc (ura4 $\left.{ }^{+}\right)$ & this study \\
\hline FG2157 & $\mathrm{h}^{-}$his7-366 atf1-12 myc (ura4+) pcrl::his7 ${ }^{+}$ & this study \\
\hline FG2158 & $\mathrm{h}^{+}$his7-366 spc1-12 myc (ura4+) pcr1::his7 ${ }^{+}$ & this study \\
\hline FG2201 & $\begin{array}{l}\mathrm{h}^{-} / \mathrm{h}^{+} \text {ade6-M210/ade6-M216 his7-366/his7-366 spc1 HA6H } \\
\text { (ura4 }{ }^{+} / \mathrm{spc1-12} \text { myc (ura4 }{ }^{+} \text {) }\end{array}$ & this study \\
\hline FG2202 & $\begin{array}{l}\text { h-/h+ ade6-M210/ade6-M216 his7-366/his7-366 spc1 HA6H } \\
\left(\text { ura }^{+}\right) / \text {spc1AY-12 myc (ura4+) }\end{array}$ & this study \\
\hline FG2203 & $\begin{array}{l}\mathrm{h}^{-} / \mathrm{h}^{+} \text {ade6-M210/ade6-M216 his7-366/his7-366 spc1 HA6H } \\
\left.\left(\text { ura }^{+}\right) / \text {spc1TF-12 myc (ura4 }{ }^{+}\right)\end{array}$ & this study \\
\hline FG2204 & $\begin{array}{l}\text { h-/h+ ade6-M210/ade6-M216 his7-366/his7-366 spc1 HA6H } \\
\left(\text { ura }^{+}\right) / \text {spc1AF-12 myc (ura4 }\end{array}$ & this study \\
\hline
\end{tabular}

All strains are leu1-32 ura4-D18.

dium (YES) and synthetic minimal medium $\left(\mathrm{EM} \mathrm{M}_{2}\right)$ were used in growing $S$. pombe cells.

\section{Construction of the myc-tagged alleles}

To introduce an epitope tag at the carboxyl terminus of the proteins, the pRIP-12myc vector was designed as follows. A cassette containing 12 tandem copies of the myc epitope was amplified by PCR using a 5' primer containing a Kpnl site. This DNA fragment was cloned into the Smal site of the pRIP42 vector (Basi et al. 1993). The resultant plasmid was digested with Pstl and Ndel and religated to eliminate the nmtl promoter sequence, producing pRIP-12myc. Sequences encoding the carboxyl terminus of different proteins were amplified by PCR and cloned in this vector as BamHI or BamHI-Kpnl fragments, except for the atf1 fragment, which was cloned as a BgllI-Kpnl fragment. The foll lowing ol igonucleotides were used: spc1 5' primer CGCGGATCCATGGCAGAATTTATTCGTACA-C and $3^{\prime}$ primer CGGGGTACCTTGGATTGCAGTTCATTATCCATG; wisl $5^{\prime}$ primer GGTCAGACTTGGCAGATCTACGTCCAG and 3' primer CGGGGTACCTTGCTTCTTTTTTCACСТTTCTCTTTAAGAGCG; atf1 5' primer GGAAGATCTATGTCCCCGTCTCCCGTCAATACTTCC and $3^{\prime}$ primer CGGGGTACCACGTACCCTAAATTGATTCTTTG$A G C$. The resultant plasmids were used for integrative transformation of wild-type cells (PR109) after linearization at the Nrul site of spcl, the Hpal site of wisl, or the Aflll site of atfl.

\section{Construction of spc mutants}

Mutations in the spcl sequence were made as follows. The $5^{\prime}$ end of spcl (codons 1-177) was amplified by PCR with the $5^{\prime}$ primer used for the myc-tagged constructs and the mutagenic $3^{\prime}$ primers: CGAGTAGAAACATAGCCAGCCATTTGAGG for the T171A mutant; CGAGTAGAAAC $\overline{G A A}$ GCCCGTCATTTGAGG for the Y173F mutant; and CGAGTAGAAACGAAGCCAGCCATTTGAGG for the T171A Y173F double mutant. The underlined sequence indicates the mutated codons. The $3^{\prime}$ end of spc1 (codons 168-341) was amplified with the $3^{\prime}$ primer used for the myc-tagged construct and the mutagenic $5^{\prime}$ primers: CCTCAAATGGCTGGCTATGTTTCTACTCG for the T171A mutant; CCTCAAATGACGGGCTTCGTTTCTACTCG for the Y173F mutant; and CCTCAAATGGCTGGCTTCGTTTCTACTCG for the T171A Y173F double mutant. Then, for each mutant, the $5^{\prime}$ and $3^{\prime}$ PCR products were mixed in equal ratios and amplified with the $5^{\prime}$ and $3^{\prime}$ primers used for the myc-tagged constructions. The mutated spcl sequences were then subcloned in the pRIP-12myc plasmid after digestion with BamHI and $\mathrm{Kpnl}$ and sequenced. The resulting plasmids were then linearized with Bglll and used to transform wild-type cells (PR110).

\section{Stress treatment of cells}

Cells were grown to early logarithmic phase $\left(O D_{600}=\sim 0.5\right)$ at $30^{\circ} \mathrm{C}$. High-osmolarity stress was achieved by adding a onethird volume of prewarmed medium containing $2.4 \mathrm{M} \mathrm{KCl}$ to the culture to obtain a final $\mathrm{KCl}$ concentration of $0.6 \mathrm{M}$. All experiments used YES medium unless otherwise indicated. For the immunoblotting analysis, cells were harvested by filtration and immediately frozen in liquid nitrogen.

Immunoblotting of Spc1

Cells $\left(10 \mathrm{OD}_{600}\right)$ were lysed in $0.2 \mathrm{ml}$ of lysis buffer $(50 \mathrm{~mm}$ Tris at $\mathrm{pH} 8.0,150 \mathrm{~mm} \mathrm{NaCl}, 5 \mathrm{~mm}$ EDTA, $10 \%$ glycerol, $50 \mathrm{~mm}$ $\mathrm{NaF}, 1 \mathrm{~mm} \mathrm{Na} \mathrm{VO}_{4}, 1 \mu \mathrm{g} / \mathrm{ml}$ each of leupeptin, aprotinin, and pepstatin, $1 \mathrm{~mm}$ PM SF) by breaking cells with glass beads under vigorous agitation. After centrifugation $(10,000 x$ for $10 \mathrm{~min})$, the protein concentration in the supernatant was estimated by measuring $A_{280}$. A bout $150 \mu \mathrm{g}$ of total protein was loaded for each sample and resolved by SDS-PAGE. The tyrosine phosphorylation status of Spcl was determined by immunoblotting with the anti-phosphotyrosine p38 (tyr-182) MAPK antibody ( $N$ ew England Biolabs) or the anti-phosphotyrosine 4G10 (Upstate Biotechnology) antibody. The amount of Spc1-myc loaded 
was evaluated with the anti-myc $9 \mathrm{E} 10$ (BabCo) antibody. Immunoreactive bands were reveal ed with horseradish peroxidaseconjugated secondary antibodies and the SuperSignal Western blotting detection system (Pierce).

\section{Purification of Spcl under native conditions}

A bout 20 OD $_{600}$ cells of strain FG2150, bearing a chromosomal copy of spc1 encoding Spcl tagged with two HA epitopes and 6 histidine residues, and a chromosomal copy of wisl encoding Wisl protein tagged with 12 myc were harvested and lysed in lysis buffer containing $50 \mathrm{~mm}$ Tris at $\mathrm{pH} 8.0,150 \mathrm{~mm} \mathrm{NaCl}, 5$ mM EGTA, $10 \mathrm{~mm}$ imidazole, 0.2\% N P-40, 10\% glycerol, $50 \mathrm{~mm}$ $\mathrm{NaF}, 1 \mathrm{~mm} \mathrm{Na} \mathrm{VO}_{4}, 1 \mu \mathrm{g} / \mathrm{ml}$ each of leupeptin, aprotinin, and pepstatin, and $1 \mathrm{~mm}$ PMSF. After centrifugation, the supernatants were incubated with $\mathrm{Ni}^{2+}$-NTA-agarose beads, resolved by SDS-PAGE, and the purified proteins were detected by immunoblotting with either the anti-HA 12CA5 antibody for Spc1 or the anti-myc 9E10 antibody for Wis1.

\section{Indirect immunofluorescence microscopy}

Mid-log phase cells were harvested by filtration on glass-fiber filters and fixed by immersion in $-80^{\circ} \mathrm{C}$ methanol for $10 \mathrm{~min}$. After three washes in PEM buffer (100 mM PIPES, 1 mM EGTA, $1 \mathrm{~mm} \mathrm{MgSO}{ }_{4}$ at $\mathrm{pH} 6.9$ ), the cell wall was digested at $37^{\circ} \mathrm{C}$ for $10 \mathrm{~min}$ with $0.5 \mathrm{mg} / \mathrm{ml}$ Zymolyase 100T (Seikagaku America, Rockville, MD) in PEM S buffer (PEM buffer supplemented with $1 \mathrm{M}$ sorbitol), followed by permeabilization with $1 \%$ Triton $\mathrm{X}$ 100. After washes, cells were blocked for $30 \mathrm{~min}$ at room temperature in PEM BAL buffer (PEM buffer at $\mathrm{pH} 6.9$ supplemented with $1 \% \mathrm{BSA}, 0.1 \% \mathrm{NaN}_{3}, 100 \mathrm{~mm}$ L-lysine monohydrochloride). Primary antibodies, anti-myc 9E10 antibody (BabCo), and anti-GST antibody were added to PEMBAL and incubated overnight at room temperature. After washing three times in PEM BAL, cells were incubated for $5 \mathrm{hr}$ at room temperature in PEM BAL containing CY 3-conjugated anti-mouse IgG (Jackson Laboratories) or FITC-conjugated anti-rabbit IgG (Zymed) as secondary antibodies. Cells were photographed using a Nikon Eclipse E800 microscope equipped with a Photometrics Quantix CCD camera. Images were acquired with IPLab Spectrum software (Signal Analytics Corporation).

\section{Acknowledgments}

We thank Odile M ondesert for providing expert technical assistance and the Scripps Cell Cycle groups for support and encouragement. F.G. was supported by the Leukemia Society of America, G.D. was supported by an American Cancer Society International Cancer Research Fellowship, and K.S. was supported by a California Division-American Cancer Society Fellowship. This research was supported by $\mathrm{N}$ ational Institutes of Health grant GM 41281 awarded to P.R.

The publication costs of this article were defrayed in part by payment of page charges. This article must therefore be hereby marked "advertisement" in accordance with 18 USC section 1734 solely to indicate this fact.

\section{References}

Alfa, C., P. Fantes, J. Hyams, M. M cLeod, and E. Warbrick. 1993. Experiments with fission yeast. Cold Spring Harbor Laboratory Press, Cold Spring Harbor, NY.

Basi, G., E. Schmid, and K. Maundrell. 1993. TATA box mutations in the Schizosaccharomyces pombe nmt1 promoter af- fect transcription efficiency but not the transcription start point or thiamine repressibility. Gene 123: 131-136.

Canagarajah, B.J., A. Khokhlatchev, M.H. Cobb, and E.J. Goldsmith. 1997. Activation mechanism of the MAP kinase ERK2 by dual phosphorylation. Cell 90: 859-869.

Cano, E. and L.C. Mahadevan. 1995. Parallel signal processing among mammalian MAPKs. Trends Biochem. Sci. 20: 117122.

Cavigelli, M., F. Dolfi, F.X. Claret, and M. Karin. 1995. Induction of c-fos expression through JNK-mediated TCF/Elk-1 phosphorylation. EMBO J. 14: 5957-5964.

Chen, R.-H., C. Sarnecki, and J. Blenis. 1992. N uclear localization and regulation of erk- and rsk-encoded protein kinases. Mol. Cell. Biol. 12: 915-927.

Cobb, M.H. and E.J. Goldsmith. 1995. How MAP kinases are regulated. J. Biol. Chem. 270: 14843-14846.

Davis, R.J. 1994. MAPKs: N ew JNK expands the group. Trends Biochem. Sci. 19: 470-473.

Degols, G., K. Shiozaki, and P. Russell. 1996. Activation and regulation of the Spcl Mitogen-activated protein kinase in Schizosaccharomyces pombe. Mol. Cell. Biol. 16: 28702877.

Derijard, B., M. Hibi, H. Wu, T. Barrett, B. Su, T. Deng, M. Karin, and R.J. Davis. 1994. JNK1: A protein kinase stimulated by UV light and $\mathrm{Ha}$-Ras that binds and phosphorylates the c-jun Activation domain. Cell 76: 1025-1037.

Fukuda, M., I. Gotoh, Y. Gotoh, and E. N ishida. 1996. Cytoplasmic localization of mitogen-activated protein kinase kinase directed by its $\mathrm{NH}_{2}$-terminal, leucine-rich short amino acid sequence, which acts as a nuclear export signal. J. Biol. Chem. 271: 20024-20028.

Fukuda, M., Y. Gotoh, and E. N ishida. 1997. Interaction of M AP kinase with MAP kinase kinase: its possible role in the control of nucleoplasmic transport of MAP kinase. EMBO J. 16: 1901-1908.

Gonzalez, F.A., A. Seth, D.L. Raden, D.S. Bowman, F.S. Fay, and R.J. Davis. 1993. Serum-induced translocation of mitogenactivated protein kinase to the cell surface ruffling membrane and the nucleus. J. Cell Biol. 122: 1089-1101.

Han, J., J.-D. Lee, L. Bibbs, and R.J. Ulevitch. 1994. A MAP kinase targeted by endotoxin and hyperosmolarity in mammalian cells. Science 265: 808-811.

Hill, C.S. and R. Treisman. 1995. Transcriptional regulation by extracellular signals: Mechanisms and specificity. Cell 80: 199-211.

Kallunki, T., B. Su, I. Tsigelny, H. Sluss, B. Derijard, G. Moore, R. Davis, and M. Karin. 1994. JN K2 contains a specificitydetermining region responsible for efficient c-Jun binding and phosphorylation. Genes \& Dev. 8: 2996-3007.

Karin, M. 1994. Signal transduction from the cell surface to the nucleus through the phosphorylation of transcription factors. Curr. Opin. Cell Biol. 6: 415-424.

Karin, M. and T. Hunter. 1995. Transcriptional control by protein phosphorylation: Signal transmission from the cell surface to the nucleus. Curr. Biol. 5: 747-757.

Kato, T., K. Okazaki, H. Murakami, P. Stettler, P. Fantes, and H. Okayama. 1996. Stress signal, mediated by a HOG1-like MAP kinase controls sexual development in fission yeast. FEBS Lett. 378: 207-212.

Kyriakis, J., P. Banerjee, E. Nikolakaki, T. Dai, E. Rubie, M. A hmad, J. Avruch, and J. Woodgett. 1994. The stress-activated protein kinase subfamily of c-Jun kinases. Nature 369: 156-160.

Lenormand, P., C. Sardet, G. Pagès, G. L'Allemain, A. Brunet, and J. Pouysségur. 1993. Growth factors induce nuclear transl ocation of MAP kinases (p42 ${ }^{\text {mapk }}$ and $p 44^{\text {mapk }}$ ) but not 
of their activator MAP kinase kinase (p45 mapkk) in fibroblasts. J. Cell Biol. 122: 1079-1088.

Marshall, C.J. 1994. MAP kinase kinase kinase, MAP kinase kinase and MAP kinase. Curr. O pin. Genet. Dev. 4: 82-89.

Millar, J.B.A., V. Buck, and M.G. Wilkinson. 1995. Pypl and Pyp2 PTPases dephosphorylate an osmosensing M AP kinase controlling cell size at division in fission yeast. Genes \& Dev. 9: 2117-2130.

Mitchison, J.M. 1970. Physiological and cytological methods for Schizosaccharomyces pombe. Methods Cell Physiol. 4: 131146.

Nishida, E. and Y. Gotoh. 1993. The MAP kinase cascade is essential for diverse signal transduction pathways. Trends Biochem. Sci. 18: 128-131.

Raingeaud, J., S. Gupta, J. Rogers, M. Dickens, J. Han, R. Ulevitch, and R. Davis. 1995. Pro-inflammatory cytokines and environmental stress cause p38 mitogen-activated protein kinase activation by dual phosphorylation on tyrosine and threonine. J. Biol. Chem. 270: 7420-7426.

Rouse, J., P. Cohen, S. Trigon, M.A.-L. Morange, D. Zamanillo, T. Hunt, and A. N ebreda. 1994. A novel kinase cascade triggered by stress and heat shock that stimulates MAPKAP kinase 2 and phosphorylation of the small heat shock proteins. Cell 78: 1027-1037.

Samejima, I., S. M ackie, and P.A. Fantes. 1997. Multiple modes of activation of the stress-responsive MAP kinase pathway in fission yeast. EMBO J. 16: 6162-6170.

Sanghera, J.S., M. Peter, E.A. Nigg, and S.L. Pelech. 1992. Immunological characterization of avian MAP kinases: Evidence for nuclear localization. Mol. Biol. Cell 3: 775-787.

Seger, R. and E.G. Krebs. 1995. The MAPK signaling cascade. FASEB J. 9: 726-735.

Seth, A., F.A. Gonzalez, S. Gupta, D.L. Raden, and R.J. Davis. 1992. Signal transduction within the nucleus by mitogen activated protein kinase. J. Biol. Chem. 267: 24796-24804.

Shiozaki, K. and P. Russell. 1995. Cell-cycle control linked to extracellular environment by MAP kinase pathway in fission yeast. Nature 377: 739-743.

- - - 1996. Conjugation, meiosis, and the osmotic stress response are regulated by Spcl kinase via Atf1 transcription factor in fission yeast. Genes \& Dev. 10: 2276-2288.

Shiozaki, K., M. Shiozaki, and P. Russell. 1997. Mcs4 mitotic catastrophe suppressor regulates the fission yeast cell cycle through the Wik1-Wisl-Spc1 kinase cascade. Mol. Biol. Cell 8: 409-419.

Wang, Z., P.C. Harkins, R.J. Ulevitch, J. Han, M.H. Cobb, and E.J. Goldsmith. 1997. The structure of mitogen-activated protein kinase p38 at 2.1 A resolution. Proc. Natl. Acad. Sci. 94: 2327-2332.

Waskiewicz, A.J. and J.A. Cooper. 1995. Mitogen and stress response pathways: MAP kinase cascades and phosphatase regulation in mammals and yeast. Curr. Opin. Cell Biol. 7: 798-805.

Watanabe, Y. and M. Yamamoto. 1996. Schizosaccharomyces pombe $\mathrm{prr}^{+}$encodes a CREBS/ATF protein in regulation of gene expression for sexual development. Mol. Cell. Biol. 16: 704-711.

Wilkinson, M.G., M. Samuels, T. Takeda, W.M. Toone, J.-C. Shieh, T. Toda, J.B.A. Millar, and N. Jones. 1996. The Atf1 transcription factor is a target for the Sty 1 stress-activated MAP kinase pathway in fission yeast. Genes \& Dev. 18: 2289-2301.

Zheng, C.F. and K.L. Guan. 1994. Cytoplasmic localization of the mitogen-activated protein kinase activator MEK. J. Biol. Chem. 269: 19947-19952. 


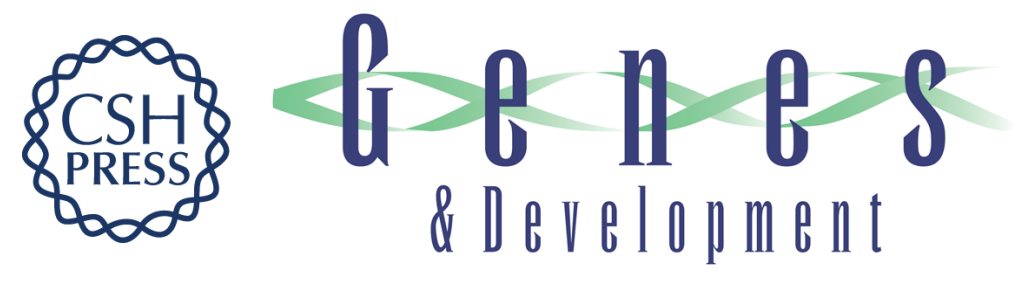

\section{Phosphorylation and association with the transcription factor Atf1 regulate localization of Spc1/Sty1 stress-activated kinase in fission yeast}

Frédérique Gaits, Geneviève Degols, Kazuhiro Shiozaki, et al.

Genes Dev. 1998, 12:

Access the most recent version at doi:10.1101/gad.12.10.1464

\section{References This article cites 37 articles, 19 of which can be accessed free at: http://genesdev.cshlp.org/content/12/10/1464.full.html\#ref-list-1}

\section{License}

Email Alerting Service 